\title{
Effect of surgical intraocular pressure lowering on retinal structures - nerve fibre layer, foveal avascular zone, peripapillary and macular vessel density: 1 year results
}

\author{
Tun Wang Ch'ng $\mathbb{1}^{1,2} \cdot$ Kevin Gillmann $\mathbb{1}^{1} \cdot$ Kirsten Hoskens $^{1} \cdot$ Harsha L. Rao $\mathbb{\circledR}^{3} \cdot$ André Mermoud ${ }^{1} \cdot$ \\ Kaweh Mansouri ${ }^{1,4}$
}

Received: 17 December 2018 / Revised: 16 June 2019 / Accepted: 11 July 2019 / Published online: 13 August 2019

(c) The Author(s), under exclusive licence to The Royal College of Ophthalmologists 2019

\begin{abstract}
Objectives To determine the effect of surgical intraocular pressure (IOP) lowering on peripapillary retinal nerve fibre layer thickness (RNFL), fovea avascular zone (FAZ), peripapillary and macular vessel density (VD) in glaucoma using with optical coherence tomography angiography (OCT-A).

Methods This was a prospective observational study performed at the Glaucoma Research Centre, Montchoisi Clinic, Lausanne. In total 40 eyes with open-angle glaucoma were included. OCT-A scans were performed before glaucoma surgery, and at 1-month, 3-month, 6-month, and 12-month post-operatively. AngioVue AngioAnalytic (Optovue Inc, Fremont, CA) software was used to analyse the RNFL, FAZ, peripapillary and macular VD. Changes were analysed using analysis of variance (ANOVA) models.

Results Mean IOP dropped from $19.4( \pm 7.0) \mathrm{mmHg}$ pre-surgery and stabilized at $13.0( \pm 3.1) \mathrm{mmHg}$ at 12 months $(p<$ $0.001)$. The number of topical medications reduced from $2.2( \pm 1.2)$ preoperatively to $0.4( \pm 0.8)$ at 1 year $(p<0.001)$. Peripapillary RNFL thickness was transiently increased at 1 -month postoperatively $(p=0.03)$. Peripapillary VD fluctuated throughout the follow-up. Foveal VD showed increased perfusion at 3 and 6 months post-operatively with minimal changes at 1 month $(p<0.01)$. Glaucoma surgery had a significant effect initially on FAZ area $(p=0.03)$, FAZ perimeter $(p=0.02)$ and Acircularity Index (AI) $(p=0.04)$. By 12-months FAZ measurements had reversed to baseline values.

Conclusions Peripapillary and macular microcirculations responded differently to surgically induced IOP reduction. Peripapillary microcirculation was IOP-independent within the studied range of surgically-controlled IOP. Macular microcirculation, however, exhibited a "delayed response" followed by near-normal reperfusion after glaucoma surgery. FAZ parameters could be potentially useful modalities to assess vascular reperfusion after glaucoma surgery as, amongst all studied parameters, the area was the most sensitive to surgically induced IOP changes.
\end{abstract}

Kaweh Mansouri

kwmansouri@gmail.com

1 Glaucoma Research Center, Montchoisi Clinic, Swiss Visio Network, Lausanne, Switzerland

2 Department of Ophthalmology, Hospital Kuala Lumpur, Kuala Lumpur, Malaysia

3 Narayana Nethralaya, Bangalore, India

4 Department of Ophthalmology, University of Colorado School of Medicine, Denver, CO, USA

\section{Introduction}

Elevated intraocular pressure (IOP) is the main risk factor in the pathogenesis of glaucoma [1-8] and IOP-lowering is the mainstay of treatment to prevent its progression [9]. However, it is not uncommon to see cases of glaucoma progressing despite a strict control of IOPs within individualised target ranges.

Impaired microvascular circulation has been shown in glaucomatous eyes [10-12]. Various studies have shown that ocular perfusion plays a role in the progression of glaucoma $[13,14]$. Techniques to assess ocular vasculature were limited to research settings till now and had various limitations. Invention of Optical Coherence Tomography Angiography (OCT-A) enabled clinicians to assess ocular 
microvasculature without injection of contrast into the human body, thus improving the safety profile of such investigations. OCT-A detects red blood cells (RBCs) motion within the blood vessels and uses the differences between B-scans acquired at same locations to generate contrast associated with motion [15]. It produces high resolution images of retinal microvasculature [15]. It is possible to assess the vasculature of optic nerve head and macula in detail as well as delineate the fovea avascular zone (FAZ) using OCT-A in clinics now. Furthermore, Spectral Domain OCT (SD-OCT) has been shown to effectively assess peripapillary retinal fibre layer (RNFL) thickness in glaucoma [16, 17].

Surgical IOP-lowering has been shown to cause changes in ocular haemodynamics [18-22]. Studies have indicated that ocular blood flow increases after significant drop of IOP post glaucoma surgery [23]. However, to date, little is still known about how this specifically affects ocular microvasculature. In this study, we sought to determine the nature and the extent of changes in peripapillary and macular microvasculature, and in the FAZ post glaucoma surgery. To the best of our knowledge, this is the first study to describe the progression of microvascular changes over a period of 1 year following glaucoma surgery.

\section{Materials and methods}

This was a prospective observational study performed at the Glaucoma Research Centre, Montchoisi Clinic, Swiss Visio Network, Lausanne. The study was approved by the local institutional review board and adhered to the tenants of the Declaration of Helsinki. Written informed consent was obtained from each patient prior to their enrolment in the study. (ClinicalTrials.gov identifier: NCT02757677)

\section{Study population}

All patients with open angle glaucoma (OAG) undergoing glaucoma surgery at the investigation centre between December 2015 and November 2016 were prospectively enrolled when they matched the inclusion criteria. Inclusion criteria were (1) clinically diagnosed glaucomatous optic disc defined by the presence of neuroretinal rim (NRR) thinning; (2) localised or diffuse RNFL defects typical for glaucoma damage observed by slit lamp examination; (3) elevated IOP over individual targets despite topical antiglaucoma medication requiring surgical intervention; and (4) 2 consecutive reproducible and reliable abnormal standard automated visual field results (Octopus, Haag Streit, Koeniz, Switzerland). Gonioscopy was performed on all recruited patients to exclude angle closure. Eyes with previous ocular surgery (except uncomplicated cataract surgery), high myopia, coexisting retinal pathologies and other ocular pathologies were excluded. Patients were recruited from the investigators' glaucoma clinics. Patients' demographics, best-corrected visual acuity (BCVA), IOP, central corneal thickness (CCT), visual field mean deviation (MD) and square of loss variance (sLV) were obtained at baseline. For all included eyes, OCT-A of the optic disc and macula were performed before glaucoma surgery, and repeated 1 month, 3 months, 6 months and 12 months after the procedure. One IOP measurement was performed at each visit using Goldmann applanation tonometry. All measurements were performed by ophthalmologists who were not involved in the study design and data collection.

\section{OCT-A image acquisition and processing}

The Avanti SD-OCT device (RTVue XR Avanti System; Optovue Inc, Fremont, CA, USA) with AngioVue AngioAnalytic software was used for the study. It uses a Split-spectrum Amplitude Decorrelation Angiography (SSADA) algorithm [24] to capture the dynamic motion of RBCs and generate a high-resolution three-dimensional (3D) image of perfused retinal vasculature [15]. OCT-A scans were acquired by trained and experienced technicians. AngioAnalytic scans in the current study consisted of 3D Angio Disc $4.5 \times 4.5 \mathrm{~mm}$, 3D Angio Retina $3.0 \times 3.0 \mathrm{~mm}$ and 3D Angio Retina $6.0 \times 6.0 \mathrm{~mm}$ scans [25]. The software (v2017.1) provides information on vessel density (VD), peripapillary RNFL thickness and parameters of FAZ. VD analysis computes the percentage of area occupied by OCTA-detected vasculature. The peripapillary region was divided into 12 sectors for RNFL thickness and 8 sectors for VD, and measurements were made in each sector separately (Fig. 1). The macular region was divided into 3 zones: fovea, parafovea and perifovea. The parafoveal and perifoveal zones were further divided in 4 quadrants and 2 hemispheres (Fig. 1). The FAZ was automatically mapped by the software. Its surface area was measured in $\mathrm{mm}^{2}$ and its perimeter in $\mathrm{mm}$. The Acircularity Index (AI) was calculated as the ratio of the FAZ perimeter to the perimeter of a circle with an area equal to that of the FAZ. Two trained and independent readers reviewed all the images. Images with poor quality were excluded. In general, images were excluded based on 1 or more of the following criteria: (1) motions artefacts, (2) poor clarity obscuring view of vasculature, (3) low signal strength index (SSI less than 47) [25], or (4) segmentation errors.

\section{Statistical Analysis}

Statistical analysis was performed with a commercially available software (Stata, version 13.1; StataCorp, College Station, TX). $P$-value of $<0.05$ was considered statistically 


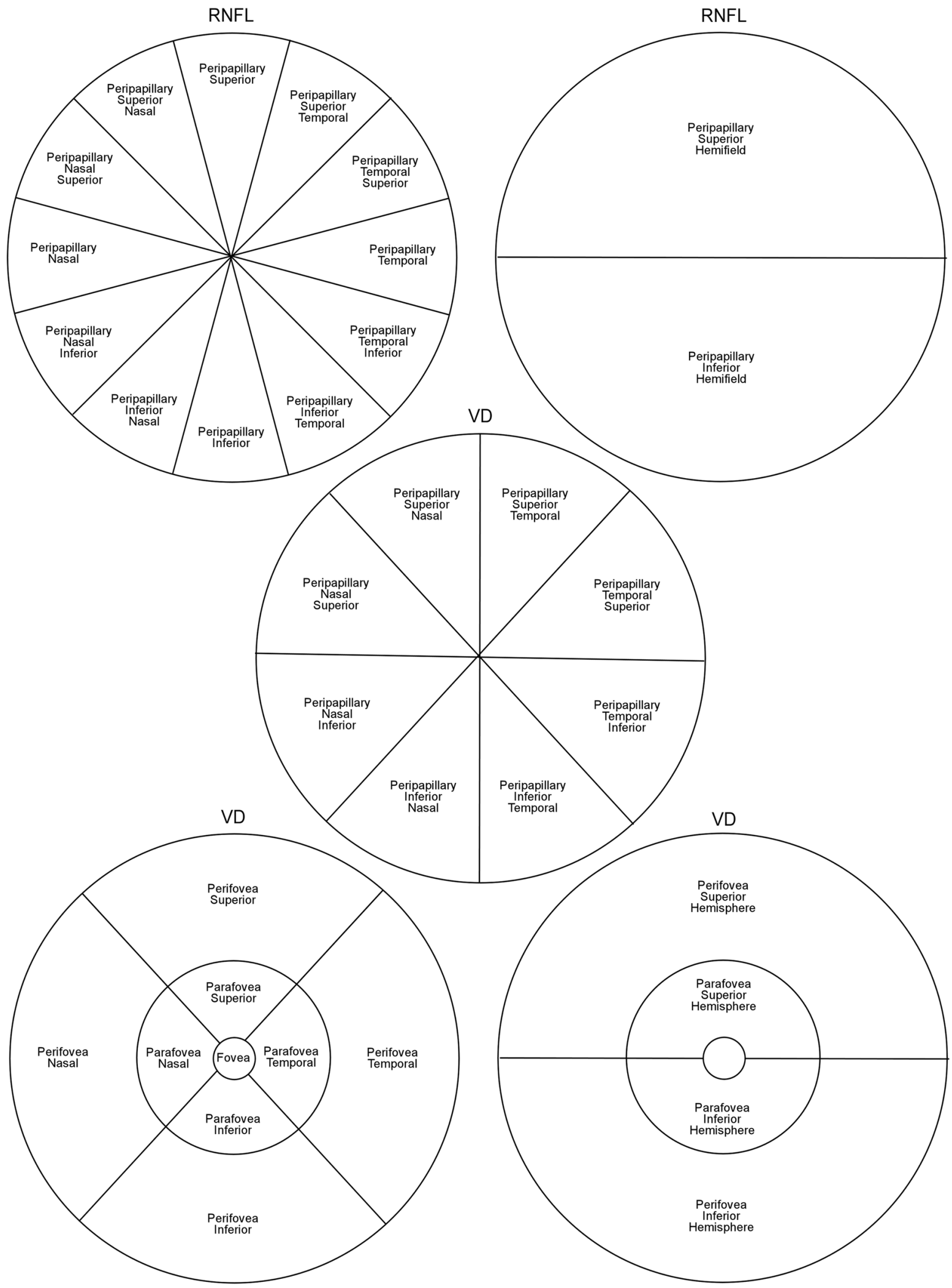

Fig. 1 Shows the delimitation of scan regions for peripapillary retinal nerve fibre layer (RNFL, top), peripapillary vessel density (VD, middle) and macular vessel density (VD, bottom) scans

significant. Quantitative data were described by mean and standard deviation (SD). Analysis of variance (ANOVA) models were used to compare RNFL thickness, VD measurement of peripapillary, macular regions and FAZ 


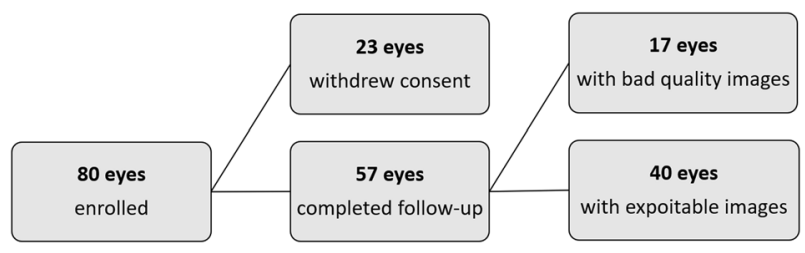

Fig. 2 A flowchart of the excluded eyes following study enrolment

measurements at various scheduled visits to evaluate the changes following glaucoma surgery. Kruskal Wallis tests were used to confirm the results for non-parametric data. Posthoc tests were used for pairwise comparisons of measurements between the visits.

\section{Results}

A total of 80 open-angle glaucoma eyes from 80 patients were enrolled in the study. Seventeen eyes of 17 patients were excluded due to low quality images (SSI $<47$ and motion artefacts), and 23 withdrew their consent during the follow-up. In total, 40 eyes with good quality OCT-A scans and complete follow-up were included in the final analysis (Fig. 2). All recruited eyes underwent glaucoma surgery to achieve their desired target IOP. Mean IOP dropped at the first postoperative visit and remained stable through to 12 months (Fig. 3a). The mean percentage reduction for the cohort were $37.6 \pm 34.8 \%$ at 1 month, $27.0 \pm 30.6 \%$ at 3 months, $24.6 \pm 31.2 \%$ at 6 months and $30.5 \pm 22.8 \%$ at 12 months. At 1 year, an average of $0.4 \pm 0.8$ topical medications were required to sustain a mean IOP of $13.0 \pm$ $3.1 \mathrm{mmHg}$ (Fig. 3b). Table 1 shows a full summary of the demographic data and clinical characteristics.

\section{RNFL thickness}

There were significant changes in mean RNFL thickness immediately following glaucoma surgery. We observed a transient increase in peripapillary RNFL thickness at 1month postoperatively, which subsequently reverted to presurgery levels at 3-month and remained stable through the rest of the follow-up. This transient increase was statistically significant in both superior and inferior hemifields (Fig. 3c, Table 2).

\section{Vessels densities (VD)}

\section{Peripapillary VD}

Peripapillary VD fluctuated widely throughout the followup, remaining under the mean baseline value. The lowest VD in the papillary area was reached at 1-month postoperatively. Values subsequently fluctuated through the a.
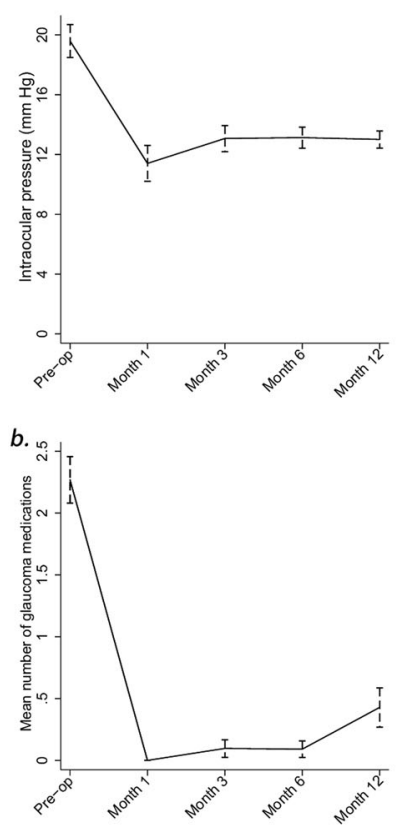

c.

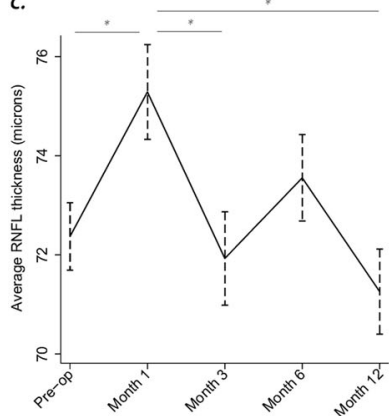

d.

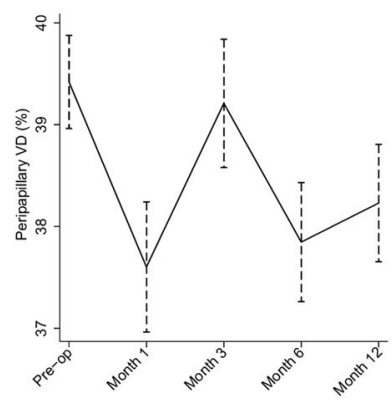

h.

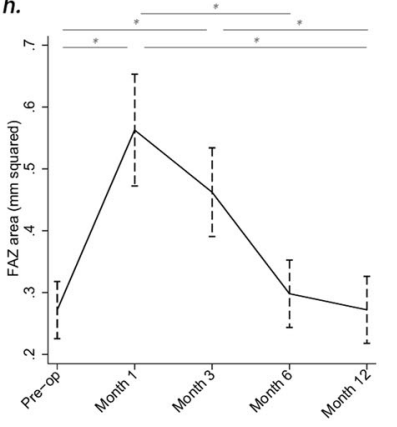

12-month follow-up. Inferior hemifield, specifically the nasal-inferior and inferior-temporal sectors, were the only areas showing statistically significant changes (Fig. 3d, Table 2).

\section{Superficial macular VD}

Superficial macular VD showed minimal changes during the first month post glaucoma surgery. After an initial 
Fig. 3 a shows the mean intraocular pressures preoperatively and at 1 month, 3 months, 6 months and 12 months after surgery. b shows the mean number of topical anti-glaucoma medications per patient, preoperatively and at 1 month, 3 months, 6 months, and 12 months after surgery. c shows mean retinal nerve fibre layer (RNFL) thickness preoperatively and at 1 month, 3 months, 6 months, and 12 months after surgery. $\mathbf{d}$ shows peripapillary vessel density (VD) preoperatively and at 1 month, 3 months, 6 months, and 12 months after surgery. e shows foveal VD preoperatively and at 1 month, 3 months, 6 months, and 12 months after surgery. $\mathbf{f}$ shows the parafoveal vessel density (VD) preoperatively and at 1 month, 3 months, 6 months, and 12 months after surgery. $\mathrm{g}$ shows the perifoveal vessel density (VD) preoperatively and at 1 month, 3 months, 6 months, and 12 months after surgery. $\mathbf{h}$ shows the fovea avascular zone (FAZ) surface preoperatively and at 1 month, 3 months, 6 months, and 12 months after surgery. Vertical dotted lines represent standard error of means. Asterisks represent statistically significant inter-visit differences $(p<$ 0.05). ANOVA showed no statistically significant variations of the parameters where no asterisks are present

reduction at 1 month, the foveal VD increased steadily through 6 months, before dropping slightly at 12 months (Fig. 3e-g, Table 2, $p<0.05$ ). VD in the parafoveal and the perifoveal regions followed the same trend with a delayed increase from 3 months, but none of the variations in these regions were statistically significant. No statistically significant association was found between any measured VD and IOP reduction or SSI $(p>0.05)$.

\section{Fovea Avascular Zone (FAZ)}

Significant changes were noted in FAZ region. We observed an increase in the size of the FAZ area and perimeter at 1-month, followed by a gradual reduction from the $3^{\text {rd }}$ month reverting back to baseline sizes at 1 year. The AI increased immediately in the post-operative period, and maintained its elevated level through to 6-months. At one year, the AI was close to baseline level. All the changes in FAZ region were statistically significant (Fig. 3h, Table 2).

No statistically significant associations were found between RNFL and VD measurement changes and the changes in IOP or SSI values $(p>0.05)$.

\section{Discussion}

\section{RNFL thickness}

Post-operative inflammation might account for the noted transient increase in RNFL thickness following glaucoma surgery. It is well known that anterior chamber inflammation last for at least 1 month after ocular surgery [26]. This has been confirmed at a molecular level, with inflammatory mediators detected in the eye for up to 1 month after intraocular surgery [27]. Such mediators, cytokines, cause blood-ocular barrier breakdown [28]. In
Table 1 Demographic and Ocular Characteristics

\begin{tabular}{|c|c|}
\hline Characteristic & Mean $\pm \mathrm{SD} ;$ No. $(\%)$ \\
\hline Age (years) & $73.1 \pm 8.1$ \\
\hline \multicolumn{2}{|l|}{ Gender } \\
\hline Male & $18(45)$ \\
\hline Female & $22(55)$ \\
\hline \multicolumn{2}{|l|}{ Laterality } \\
\hline OD & $21(53)$ \\
\hline OS & $19(47)$ \\
\hline \multicolumn{2}{|l|}{ Type of glaucoma surgery } \\
\hline Deep Sclerectomy & $19(47.5)$ \\
\hline Combine Deep Sclerectomy-Phaco & $5(12.5)$ \\
\hline Xen & $1(2.5)$ \\
\hline Combine Xen -Phaco & $13(32.5)$ \\
\hline GDD & $2(5.0)$ \\
\hline SSI & 56.9 \\
\hline \multicolumn{2}{|l|}{ Type of glaucoma } \\
\hline POAG & $25(62.5)$ \\
\hline PXG & $12(30.0)$ \\
\hline PIG & $2(5.0)$ \\
\hline Others & $1(2.5)$ \\
\hline Spherical equivalent (D) & $-0.24 \pm 2.6$ \\
\hline \multicolumn{2}{|l|}{ Mean IOP (mmHg) } \\
\hline Baseline & $19.4 \pm 7.0$ \\
\hline 12 months & $13.0 \pm 3.1$ \\
\hline \multicolumn{2}{|c|}{$\begin{array}{l}\text { Mean number of anti-glaucoma medications } \\
(n)\end{array}$} \\
\hline Baseline & $2.2 \pm 1.2$ \\
\hline 12 months & $0.4 \pm 0.8$ \\
\hline \multicolumn{2}{|l|}{ Mean visual acuity (decimal) } \\
\hline Baseline & $0.73 \pm 0.24$ \\
\hline 12 months & $0.79 \pm 0.22$ \\
\hline \multicolumn{2}{|l|}{ Mean deviation (dBs) } \\
\hline Baseline & $-10.1 \pm 6.9$ \\
\hline 12 months & $-10.8 \pm 7.2$ \\
\hline
\end{tabular}

$O D$ Right eye, $O S$ Left eye, POAG Primary Open Angle Glaucoma, PXG Pseudoexfoliation Glaucoma, PIG Pigmentary Glaucoma, SSI Signal Strength Index, GDD Glaucoma Drainage Device

their study, Zhao et al. reported a measurable increase in macular thickness for up to 3 months after uncomplicated cataract surgery [29]. Raghu et al. studied the changes in RNFL thickness pre and post-trabeculectomy, and found a transient increase in RNFL thickness 1-week post trabeculectomy, which reverted to preoperative values after 3 months [30]. The RNFL changes had no correlation with IOP changes [30]. We observed a similar finding in our longer 12-month follow-up study. RNFL thickness increased during the first month post glaucoma surgery and normalized to preoperative thickness at month 3 , 
Table 2 Changes in retinal nerve fiber layer thickness, peripapillary and superficial macular vessel density and foveal avascular zone parameters pre- and post-glaucoma surgery. ( $p$-value represents the statistical significance of the differences in the mean measurements between the five visits as calculated by analysis of variance)

\begin{tabular}{|c|c|c|c|c|c|c|}
\hline \multirow[t]{2}{*}{ RNFL thickness $(\mu \mathrm{m})($ mean, $95 \% \mathrm{CI})$} & \multirow[t]{2}{*}{ Pre-op } & \multicolumn{4}{|l|}{ Post-op } & \multirow[t]{2}{*}{$p$-value } \\
\hline & & 1 month & 3 month & 6 month & 12 month & \\
\hline Peripapillary & $72.4(71.0,73.7)$ & $75.3(73.4,77.2)$ & $71.9(70.0,73.8)$ & $73.6(71.8,75.3)$ & $71.3(69.5,73.0)$ & 0.03 \\
\hline Superior Hemifields & $72.2(70.7,73.8)$ & $75.0(72.9,77.1)$ & $71.0(68.9,73.1)$ & $73.7(71.8,75.7)$ & $71.5(69.5,73.4)$ & 0.05 \\
\hline Inferior Hemifields & $72.5(71.1,74.0)$ & $75.6(73.6,77.6)$ & $73.0(71.0,74.9)$ & $73.4(71.5,75.2)$ & $71.0(69.2,72.8)$ & 0.02 \\
\hline Temporal & $59.2(57.3,61.2)$ & $60.5(57.7,63.2)$ & $58.1(55.4,60.8)$ & $62.0(59.5,64.5)$ & $60.1(57.6,62.5)$ & 0.29 \\
\hline Superior & $78.9(77.0,80.7)$ & $82.5(80.0,85.1)$ & $77.3(74.8,79.8)$ & $80.3(77.9,82.6)$ & $78.4(76.1,80.7)$ & 0.05 \\
\hline Nasal & $69.2(67.0,71.5)$ & $73.2(70.0,76.4)$ & $69.9(66.7,73.0)$ & $69.9(67.0,72.8)$ & $67.0(64.1,69.9)$ & 0.08 \\
\hline Inferior & $83.1(81.5,84.7)$ & $85.3(83.1,87.5)$ & $83.1(80.9,85.3)$ & $83.3(81.3,85.3)$ & $81.1(79.1,83.1)$ & 0.10 \\
\hline Temporal Superior & $59.6(56.8,62.4)$ & $60.7(56.8,64.6)$ & $59.2(55.4,63.1)$ & $64.3(60.8,67.9)$ & $61.6(58.1,65.2)$ & 0.25 \\
\hline Superior Temporal & $79.2(77.1,81.3)$ & $82.2(79.2,85.1)$ & $78.2(75.3,81.1)$ & $81.4(78.7,84.0)$ & $80.3(77.7,82.9)$ & 0.29 \\
\hline Superior Nasal & $78.6(76.6,80.5)$ & $82.8(80.0,85.6)$ & $76.6(73.9,79.3)$ & $79.3(76.8,81.9)$ & $76.9(74.4,79.4)$ & 0.02 \\
\hline Nasal Superior & $72.6(70.4,74.8)$ & $75.0(71.9,78.2)$ & $71.2(68.1,74.3)$ & $71.9(69.0,74.7)$ & $69.3(66.5,72.2)$ & 0.11 \\
\hline Nasal Inferior & $65.1(62.2,67.9)$ & $71.0(67.0,75.0)$ & $68.2(64.3,72.2)$ & $67.4(63.7,71.1)$ & $64.0(60.4,67.6)$ & 0.07 \\
\hline Inferior Nasal & $82.1(80.1,84.1)$ & $84.8(82.1,87.6)$ & $82.3(79.6,85.0)$ & $82.2(80.0,84.8)$ & $79.5(77.0,82.0)$ & 0.08 \\
\hline Inferior Temporal & $84.5(82.8,86.1)$ & $85.9(83.6,88.2)$ & $84.2(82.0,86.5)$ & $84.6(82.6,86.7)$ & $83.1(81.1,85.2)$ & 0.51 \\
\hline Temporal Inferior & $58.8(57.0,60.7)$ & $60.1(57,0,60.7)$ & $56.8(54.2,59.3)$ & $59.3(57.0,61.6)$ & $58.3(55.9,60.6)$ & 0.45 \\
\hline \multirow[t]{2}{*}{ VD, \% (mean, $95 \%$ CI) } & \multirow[t]{2}{*}{ Pre-op } & \multicolumn{4}{|l|}{ Post-op } & \multirow[t]{2}{*}{$p$-value } \\
\hline & & 1 month & 3 months & 6 months & 12 months & \\
\hline Peripapillary & $39.4(38.5,40.3)$ & $37.6(36.3,38.9)$ & $39.2(38.0,40.5)$ & $37.8(36.7,39.0)$ & $38.2(37.1,39.4)$ & 0.09 \\
\hline Nasal-Superior & $36.4(35.5,37.4)$ & $34.1(32.8,35.4)$ & $35.9(34.7,37.2)$ & $35.1(34.0,36.3)$ & $35.7(34.5,36.9)$ & 0.06 \\
\hline Nasal-Inferior & $36.9(35.7,38.1)$ & $36.0(34.4,37.6)$ & $38.4(36.8,40.1)$ & $35.0(33.5,36.4)$ & $35.4(34.0,37.0)$ & 0.02 \\
\hline Inferior-Nasal & $34.9(33.3,36.5)$ & $32.5(30.3,34.7)$ & $34.9(32.7,37.0)$ & $32.2(30.2,34.2)$ & $33.9(31.9,35.9)$ & 0.15 \\
\hline Inferior-Temporal & $42.5(41.0,43.9)$ & $38.8(36.7,40.8)$ & $40.8(38.7,42.8)$ & $39.6(37.7,41.5)$ & $39.9(38.1,41.8)$ & 0.04 \\
\hline Temporal-Inferior & $45.3(43.8,46.7)$ & $43.1(41.1,45.1)$ & $44.0(42.1,46.0)$ & $44.4(42.6,46.2)$ & $43.7(41.9,45.5)$ & 0.50 \\
\hline Temporal-Superior & $46.7(45.5,47.9)$ & $45.7(43.9,47.4)$ & $46.0(44.3,47.7)$ & $46.4(44.8,48.0)$ & $46.8(45.2,48.3)$ & 0.84 \\
\hline Superior-Temporal & $39.1(37.9,40.2)$ & $37.7(36.1,39.3)$ & $40.2(38.5,41.8)$ & $38.3(36.8,39.8)$ & $38.0(36.4,39.4)$ & 0.19 \\
\hline Superior-Nasal & $35.1(33.9,36.2)$ & $34.7(33.1,36.3)$ & $34.5(32.9,36.1)$ & $33.2(31.7,34.7)$ & $33.6(32.1,35.0)$ & 0.26 \\
\hline Fovea & $25.6(23.2,27.9)$ & $24.9(21.2,28.5)$ & $30.4(27.1,33.8)$ & $31.8(29.2,34.4)$ & $27.6(24.8,30.4)$ & $<0.01$ \\
\hline Parafovea & $46.4(44.7,48.0)$ & $46.0(43.3,48.6)$ & $47.5(45.1,49.8)$ & $49.0(47.2,50.9)$ & $47.2(45.3,49.3)$ & 0.21 \\
\hline Superior-Hemisphere & $46.7(44.9,48.4)$ & $45.5(42.7,48.2)$ & $47.3(44.8,49.7)$ & $49.0(47.1,50.9)$ & $47.5(45.4,49.6)$ & 0.25 \\
\hline Inferior-Hemisphere & $46.0(44.2,47.9)$ & $46.4(43.5,49.3)$ & $47.6(45.0,50.3)$ & $49.1(47.0,51.1)$ & $47.1(44.9,49.3)$ & 0.26 \\
\hline Temporal & $46.4(44.5,48.4)$ & $47.2(44.1,50.2)$ & $49.2(46.5,51.9)$ & $49.6(47.5,51.7)$ & $47.2(44.9,49.5)$ & 0.19 \\
\hline Superior & $47.2(45.5,49.0)$ & $48.2(45.4,51.1)$ & $48.2(45.6,50.8)$ & $49.2(47.2,51.2)$ & $47.8(45.6,50.0)$ & 0.70 \\
\hline Nasal & $45.6(43.2,48.1)$ & $42.2(38.3,46.1)$ & $44.2(40.7,47.7)$ & $48.4(45.7,51.1)$ & $47.4(44.4,50.3)$ & 0.09 \\
\hline Inferior & $46.0(44.0,48.1)$ & $46.2(43.0,49.4)$ & $48.3(45.4,51.2)$ & $48.9(46.7,51.2)$ & $46.9(44.5,49.3)$ & 0.32 \\
\hline Perifovea & $43.7(42.4,45.0)$ & $43.9(41.8,46.0)$ & $45.9(43.8,47.9)$ & $45.5(43.9,47.1)$ & $43.7(42.1,45.2)$ & 0.17 \\
\hline Superior-Hemisphere & $44.5(43.1,45.9)$ & $44.1(41.9,46.2)$ & $45.7(43.5,47.8)$ & $46.0(44.5,47.6)$ & $44.1(42.5,45.7)$ & 0.32 \\
\hline Inferior-Hemisphere & $43.3(42.0,44.6)$ & $43.7(41.5,46.0)$ & $45.4(43.3,47.5)$ & $45.1(43.6,46.7)$ & $43.4(41.8,45.0)$ & 0.23 \\
\hline Temporal & $42.2(40.6,43.8)$ & $41.8(39.3,44.4)$ & $44.4(41.9,46.8)$ & $45.3(43.5,47.2)$ & $41.7(39.8,43.7)$ & 0.03 \\
\hline Superior & $43.8(42.3,45.3)$ & $42.0(39.7,44.4)$ & $44.8(42.6,47.0)$ & $45.2(43.5,46.8)$ & $43.2(41.5,45.1)$ & 0.22 \\
\hline Nasal & $47.8(46.4,49.1)$ & $48.1(45.8,50.4)$ & $48.9(46.7,51.0)$ & $49.2(47.7,50.8)$ & $47.6(46.0,49.2)$ & 0.53 \\
\hline Inferior & $42.1(40.7,43.4)$ & $42.5(40.0,44.9)$ & $44.7(42.7,46.6)$ & $44.0(42.4,45.5)$ & $42.1(40.4,43.7)$ & 0.12 \\
\hline \multirow[t]{2}{*}{ FAZ (mean, 95\% CI) } & \multirow[t]{2}{*}{ Pre-op } & Post-op & & & & \multirow[t]{2}{*}{$p$-value } \\
\hline & & 1 month & 3 month & 6 month & 12 month & \\
\hline Area, $\mathrm{mm}^{2}$ & $0.27(0.18,0.36)$ & $0.56(0.38,0.74)$ & $0.46(0.32,0.61)$ & $0.30(0.19,0.41)$ & $0.27(0.16,0.38)$ & 0.03 \\
\hline Perimeter, mm & $1.97(1.70,2.24)$ & $2.89(2.36,3.42)$ & $2.55(2.13,2.97)$ & $2.12(1.80,2.44)$ & $1.98(1.66,2.30)$ & 0.02 \\
\hline AI & $1.09(1.07,1.12)$ & $1.13(1.08,1.18)$ & $1.15(1.11,1.19)$ & $1.14(1.11,1.16)$ & $1.10(1.07,1.12)$ & 0.04 \\
\hline
\end{tabular}

RNFL Retinal nerve fibre layer, VD Vessel densities, FAZFoveal Avascular Zone, AI Acircularity index, FD FAZ Vessel Density within $300 \mu \mathrm{m}$ surrounding FAZ

which was sustained through the rest of the 1-year followup. In the literature, both post cataract and glaucoma surgery series showed similar patterns of transient increase in RNFL thickness [29, 30], strongly suggesting that the increase in RNFL thickness following surgery is independent of IOP changes. 


\section{Vessels densities (VD)}

\section{Peripapillary region}

During the follow-up period, no association between changes in peripapillary VD and changes in IOP were found in our cohort. This suggests an independence of these two factors, which is consistent with recent studies. Zeboulan et al. did not find any significant changes of peripapillary VD in their cases, with a mean baseline IOP of $23.7 \pm 9.5$ $\mathrm{mmHg}$ and a mean first month post-surgery IOP of 12.2 \pm $3.5 \mathrm{mmHg}$ ( $\geq 40 \%$ reduction) [23]. These values are similar to those of our study, with a mean baseline IOP of $19.4 \pm$ $7.0 \mathrm{mmHg}$ and a $35 \%$ IOP reduction post-surgery. On the other hand, Hollò reported an increase in peripapillary perfusion after reducing the IOP medically. His study, however, differs from the others by a significantly higher baseline IOP $(35-42 \mathrm{mmHg})$ and more marked posttreatment IOP reduction $(>50 \%)$ [31]. It is still unclear whether this observation was due to the amplitude of IOP reduction or to a potential effect of anti-glaucoma medications on VD measurements, the use of which was very limited through the post-operative period in the present study. It is, however, plausible that through an adaptative mechanism, peripapillary VD would only be affected by extremely high IOPs and wide pressure gradients. It can also be theorized that the association between IOP and VD observed by Hollò [32] and Alnawaiseh et al. [33] could be only temporary and no longer be observable 12 months after surgery or could be more significant in early glaucoma before vascular damage becomes permanent.

\section{Superficial macular region}

IOP reduction generally improves ocular blood flow [3436]. However, little is known about the long-term effect of surgically induced IOP reduction on macular VD. In OCTA imaging, the superficial inner retinal segment contains projections of the RNFL and Ganglion Cells Layers (GCL) vasculatures [24]. Normal VD in superficial fovea is $31.1 \pm$ $5.5 \%$ [37]. In our cohort, the pre-surgery superficial foveal VD was abnormally low, with a mean baseline foveal VD of $25.6 \%$ (Table 2). Considering that Ghassemi et al. calculated their mean VD value from cohort of healthy volunteers with a mean age of 36.4 years, both the age difference $(+36.7$ years $)$ and the chronic glaucoma changes intrinsically inherent to our study might explain the difference in baseline VD in our cohort. Zéboulon et al. studied the mean change of vessel density 1 month post filtering surgery and found a slight reduction of vessel density after 1 month, which was not statistically significant [23]. In our longer follow-up study, we initially observed a similar reduction of VD during the first month post-operatively, especially in central foveal region. However, we then noted a "delayed effect", in which VD increased significantly from the $3^{\text {rd }}$ month post-operatively and through to the $6^{\text {th }}$ month, reaching the healthy values described by Ghassemi et al. in their study. It then reduced gradually towards $12^{\text {th }}$ month.

Three hypotheses could explain this "delayed effect". First, it might be caused by the way glaucoma affects the auto-regulatory mechanisms of macular vasculature, as glaucoma appears to be associated with abnormal perfusion pressure and auto-regulation mechanisms [6, 38]. Furthermore, surgery-induced inflammation and transient increase in RNFL thickness could affect reperfusion.

The second postulated reason is that none of the studied eyes had exceeded their autoregulation capacities at baseline. Within the autoregulatory plateau, blood flow remains constant as well as perfusion pressures still within the autoregulatory reserve [32]. As the perfusion improves following surgery and IOP stabilization, the perfusion pressure could exceed the autoregulatory reserve and lead to an increase in blood flow. Thus, this finding might not apply to severe glaucoma, in which the autoregulatory plateau was already overcome, but further study is needed to verify this.

Finally, it is also possible that RBC flow within some foveal vessels was present but below detection threshold of the SSADA software during the initial post-operative days. This could account for the initially low VD measured by OCT-A, since a minimal flow is required by the device to detect circulating vessels [23]. This detection might also be complicated by the initial increase in RNFL thickness observed in our cases, and VD could naturally increase as the detection ability of the SSADA software improves while surgically-induced inflammation subsides.

\section{Fovea Avascular Zone (FAZ)}

The FAZ is the round capillary-free zone within the macula. It is a specialized area of the human eye for sharp and accurate central vision $[39,40]$. The normal surface area of FAZ is $0.22-0.31 \mathrm{~mm}[2,41,42]$. Recent studies found that FAZ area is enlarged in glaucoma [42, 43]. In fact, FAZ area was found to be larger in glaucoma eyes with central visual field defect compared with eyes with only peripheral visual field defect [43]. Kwon et al. observed significant correlation between FAZ area and mean visual field MD [43]. In our study, FAZ area was within the physiological range. This can be explained by the fact that all observed cases were mild-to-moderate in severity, with a mean MD of $-10.2 \mathrm{~dB}$. FAZ area and FAZ perimeter in our study significantly enlarged 1 month after glaucoma surgery and were associated with a significant increase in AI over the first 6 months post-operatively. All FAZ-associated measures normalized to baseline values at 1-year (Table 2). We 
postulate that surgical inflammation might contribute to the enlargement of FAZ in the immediate postoperative phase, as it was independent of the IOP lowering effect observed post-surgery.

\section{Limitations}

There were several limitations in our study. First, $50 \%$ of enrolled eyes could not be included in the final analysis. This is predominantly due to consent withdrawal $(28.7 \%)$, mostly explained by the high rate patients referred from distant regions or countries who did not wish to return to the investigation centre solely for research appointments. This could potentially introduce a selection bias as the remaining patients constitute a more homogenous sample with milder disease stages. Secondly, inter-visit blood pressure (BP) was not measured. BP is known to influence ocular perfusion pressure [44]. Furthermore, it is possible that antihypertensive medication in some of our hypertensive patients might have influenced retinal perfusion. Thirdly, it would have been interesting to compare these observations with a subgroup of healthy patients following routine phacoemulsification, as this would have allowed for an assessment of the retinal and peripapillary perfusion following cataract surgery to confirm which changes were specifically related to glaucoma and further assess the effect of surgery-induced ocular inflammation.

In the present study, we did not observe the same amplitude of vascular changes as other studies targeted at more advanced glaucoma patients, as the baseline IOP in our cases was still medically controlled. However, this factor is beyond our control as late presentations of advanced glaucoma are rare in our healthcare system. Analysing the relationship between the visual field defects and VD changes could potentially confirm the origin of these discrepancies. Finally, some limitations are intrinsically related to OCT-A technology: some smaller vascular changes may remain undetected as SSADA has a resolution of $18 \mu \mathrm{m}$, as compare to $5 \mu \mathrm{m}$ in full bandwidth algorithm [45]. These limitations should be taken into account when designing future studies.

\section{Conclusion}

In conclusion, peripapillary and macular microcirculations appear to respond differently to surgically induced IOP lowering. Within the physiological IOP ranges of treated glaucoma, peripapillary microcirculation fluctuates but seems to be completely IOP independent. Macular microcirculation, however, exhibits a "delayed response" of reperfusion after glaucoma surgery, allowing glaucoma patients with subnormal foveal vascularization to regain near-normal VD.
FAZ area was found to be sensitive to surgically-induced IOP changes, and significant measurement changes were observed throughout the post-operative follow-up. Thus, OCT-A measurement of VD, especially in the foveal region, could be used as a potential modality to assess vascular reperfusion and as a follow-up parameter following glaucoma surgery. Further research will be needed to determine if measurements of FAZ area and AI could potentially develop as modalities to stratify the risk of wipe-out syndrome post glaucoma surgery especially in advanced glaucoma.

\section{Summary}

What was known before:

- Glaucoma impairs macular and more generally ocular microvascular circulation.

- Macular vessel density remains reduced one month after surgical IOP reduction.

- Foveal Avascular Zone area is enlarged in glaucoma and has been correlated to visual field MD.

\section{What this study adds:}

- Macular vessel density shows a "delayed response" to surgical IOP reduction.

- Filtering surgery can restore near-normal macular vessel densities.

- Filtering surgery causes a significant transient increase in Foveal Avascular Zone area, which subsequently decreases to normal values within 12 months after surgery.

Acknowledgements This study was supported in part by the Swiss Glaucoma Research Foundation, Lausanne, Switzerland.

\section{Compliance with ethical standards}

Conflict of interest The authors declare that they have no conflict of interest.

Publisher's note: Springer Nature remains neutral with regard to jurisdictional claims in published maps and institutional affiliations.

\section{References}

1. Gordon MO, Beiser JA, Brandt JD, et al. The Ocular Hypertension Treatment Study: baseline factors that predict the onset of primary open-angle glaucoma. Arch Ophthalmol. 2002;120:714-20.

2. Kass MA, Heuer DK, Higginbotham EJ, et al. The Ocular Hypertension Treatment Study: a randomized trial determines that topical ocular hypotensive medication delays or prevents the onset 
of primary open-angle glaucoma. Arch Ophthalmol. 2002;120: 701-13.

3. The AGIS Investigators. The Advanced Glaucoma Intervention Study (AGIS): 7. The relationship between control of intraocular pressure and visual field deterioration. Am J Ophthalmol. 2000;130:429-40.

4. Leske MC, Heijl A, Hussein M, et al. Factors for glaucoma progression and the effect of treatment: the early manifest glaucoma trial. Arch Ophthalmol. 2003;121:48-56.

5. Sommer A, Katz J, Quigley HA, et al. Clinically detectable nerve fiber atrophy precedes the onset of glaucomatous field loss. Arch Ophthalmol. 1991;109:77-83.

6. Schmidl D, Garhofer G, Schmetterer L. The complex interaction between ocular perfusion pressure and ocular blood flow-relevance for glaucoma. Exp Eye Res. 2011;93:141-55.

7. Flammer J, Orgul S, Costa VP, et al. The impact of ocular blood flow in glaucoma. Prog Retin Eye Res. 2002;21:359-93.

8. Bonomi L, Marchini G, Marraffa M, et al. Vascular risk factors for primary open angle glaucoma: the Egna-Neumarkt Study. Ophthalmology. 2000;107:1287-93.

9. Heijl A, Leske MC, Bengtsson B, et al. Reduction of intraocular pressure and glaucoma progression: results from the Early Manifest Glaucoma Trial. Arch Ophthalmol. 2002;120:1268-79.

10. Akagi $T$, Iida $Y$, Nakanishi $H$, et al. Microvascular density in glaucomatous eyes with hemifield visual field defects: an optical coherence tomography angiography study. Am J Ophthalmol. 2016;168:237-249.36.

11. Lommatzsch C, Rothaus K, Koch JM, Heinz C, Grisanti S. OCTA vessel density changes in the macular zone in glaucomatous eyes. Graefes Arch Clin Exp Ophthalmol. 2018;256:1499-508.

12. Tan ACS, Tan GS, Denniston AK, Keane PA, Ang M, Milea D, et al. An overview of the clinical applications of optical coherence tomography angiography. Eye (Lond). 2018;32:262-86.

13. Gherghel D, Orgül S, Gugleta K, et al. Relationship between ocular perfusion pressure and retrobulbar blood flow in patients with glaucoma with progressive damage. Am J Ophthalmol. 2000;130:597-605.

14. Leske MC. Ocular perfusion pressure and glaucoma: clinical trial and epidemiologic findings. Curr Opin Ophthalmoll. 2009;20:73-78.

15. Rosenfeld PJ, Durbin MK, Roisman L, et al. ZEISS Angioplex spectral domain optical coherence tomography angiography: technical aspects. Dev Ophthalmol. 2016;56:18-29.

16. Chang RT, Knight OJ, Feuer WJ, et al. Sensitivity and specificity of time-domain versus spectral-domain optical coherence tomography in diagnosing early to moderate glaucoma. Ophthalmology. 2009;116:2294-9.

17. Leung CK, Cheung CY, Weinreb RN, et al. Retinal nerve fiber layer imaging with spectral-domain optical coherence tomography: a variability and diagnostic performance study. Ophthalmology. 2009;116:1257-63. 63.e1-2

18. Kuerten D, Fuest M, Koch EC, et al. Long term effect of trabeculectomy on retrobulbar haemodynamics in glaucoma. Ophthalmic Physiol Opt. 2015;35:194-200.

19. Berisha F, Schmetterer K, Vass C, et al. Effect of trabeculectomy on ocular blood flow. Br J Ophthalmol. 2005;89:185-8.

20. Januleviciene I, Siaudvytyte L, Diliene V, et al. Effect of trabeculectomy on ocular hemodynamic parameters in pseudoexfoliative and primary open-angle glaucoma patients. J Glaucoma. 2015;24:e52-e56.

21. Trible JR, Sergott RC, Spaeth GL, et al. Trabeculectomy is associated with retrobulbar hemodynamic changes. A color Doppler analysis. Ophthalmology. 1994;101:340-51.

22. Poinoosawmy D, Indar A, Bunce C, et al. Effect of treatment by medicine or surgery on intraocular pressure and pulsatile ocular blood flow in normal-pressure glaucoma. Graefes Arch Clin Exp Ophthalmol. 2002;240:721-6.
23. Zéboulon P, Léveque PM, Brasnu E, et al. Effect of Surgical Intraocular Pressure Lowering on Peripapillary and Macular Vessel Density in Glaucoma Patients: An Optical Coherence Tomography Angiography Study. J Glaucoma. 2017; 26:466-72.

24. de Carlo TE, Romano A, Waheed NK, Duker JS. A review of optical coherence tomography angiography (OCTA). Int J Retin Vitr. 2015;1:5. Published2015

25. RTVue XR Avanti User Manual International Software Version2017.1 With DualTrac P/N 580-51219-005 Rev A. Optovue, Inc 2800 Bayview Drive Fremont, CA 94538, USA.

26. Chee SP, Ti SE, Sivakumar M, et al. Postoperative inflammation: extracapsular cataract extraction versus phacoemulsification. J Cataract Refract Surg. 1999;25:1280-5.

27. Xu H, Chen M, Forrester JV, et al. Cataract surgery induces retinal pro-inflammatory gene expression and protein secretion. Invest Ophthalmol Vis Sci. 2011;52:249-55.

28. Bhagat K, Hingorani AD, Palacios M, et al. Cytokine-induced venodilatation in humans in vivo: eNOS masquerading as iNOS. Cardiovasc Res. 1999;41:754-64.

29. Zhao Z, Wen W, Jiang C, et al. Changes in macular vasculature after uncomplicated phacoemulsification surgery: optical coherence tomography angiography study. J Cataract Refract Surg. 2018;44:453-8.

30. Raghu N, Pandav SS, Kaushik S, et al. Effect of trabeculectomy on RNFL thickness and optic disc parameters using optical coherence tomography. Eye. 2012;26:1131-7.

31. Hollo Gabor. Influence of large intraocular pressure reduction on peripapillary OCT vessel density in ocular hypertensive and glaucoma eyes eyes. J Glaucoma. 2017;26:e7-e10.

32. Hollò G. Optical coherence tomography angiography in glaucoma. Turk J Ophthalmol. 2018;48:196-201.

33. Alnawaiseh M, Müller V, Lahme L, Merté RL, Eter N. Changes in flow density measured using optical coherence tomography angiography after iStent insertion in combination with phacoemulsification in patients with open-angle Glaucoma. J Ophthalmol. 2018;2018:2890357.

34. Hafez AS, RLG Bizzarro, Rivard M, et al. Changes in optic nerve head blood flow after therapeutic intraocular pressure reduction in glaucoma patients and ocular hypertensives. Ophthalmology. 2003;110:201-10.

35. Trible JR, Costa VP, Sergott RC, et al. The influence of primary open-angle glaucoma upon the retrobulbar circulation: baseline, postoperative and reproducibility analysis. Trans Am Ophthalmol Soc. 1993;91:245-61. 261-265

36. James CB. Effect of trabeculectomy on pulsatile ocular blood flow. Br J Ophthalmol. 1994;78:818-22.

37. Ghassemi F, Fadakar K, Bazvand F, et al. The quantitative measurements of vascular density and flow areas of macula using optical coherence tomography angiography in normal volunteers. Ophthalmic Surg Lasers Imaging Retin. 2017;48: 478-86.

38. Tielsch JamesM, Katz Joanne, Sommer Alfred, et al. Hypertension, perfusion pressure, and primary open-angle glaucomaa population-based assessment. Arch Ophthalmol. 1995;113: 216-21.

39. Jonas JB, Schneider U, Naumann GO. Count and density of human retinal photoreceptors. Graefes Arch Clin Exp Ophthalmol. 1992;230:505-10.

40. Yu DY, Cringle SJ, Su EN. Intraretinal oxygen distribution in the monkey retina and the response to systemic hyperoxia. Invest Ophthalmol Vis Sci. 2005;46:4728-33.

41. Ghassemi F, Mirshahi R, Bazvand F, et al. The quantitative measurements of foveal avascular zone using optical coherence tomography angiography in normal volunteers. J Curr Ophthalmol. 2017;29:293-9. 
42. Zivkovic M, Dayanir V, Kocaturk T, et al. Foveal avascular zone in normal tension glaucoma measured by optical coherence tomography angiography. Biomed Res Int. 2017;3079141:7.

43. Kwon J, Choi J, Shin JW, et al. Alterations of the foveal avascular zone measured by optical coherence tomography angiography in glaucoma patients with central visual field defects. Invest Ophthalmol Vis Sci. 2017;58:1637-45.
44. Costa VitalP, Harris Alon, Anderson Douglas, et al. Ocular perfusion pressure in glaucoma. Acta Ophthalmol. 2014;92: e252-e266.

45. Gao SimonS, Liu Gangjun, Huang David, et al. Optimization of the split-spectrum amplitude-decorrelation angiography algorithm on a spectral optical coherence tomography system. Opt Lett. $2015 ; 40: 2305-8$. 